\section{H.J. Lowe}

Section of Medical Informatics, University of Pittsburgh, Pittsburgh PA, USA

\section{Introduction}

Information systems are the foundation of many important Medical Informatics activities designed to support clinical care, facilitate biomedical research and promote life-long learning. The evolution and refinement of these systems is, therefore, of importance to all who work in the field. Currently, a number of exciting trends are emerging as biomedical information systems increasingly adopt client-server, object-oriented database models and Internet technologies to improve information representation and retrieval.

One of the most exciting developments has been the increasing use of the Internet and specifically the World Wide Web (WWW) [1] as a technology for the distribution of biomedical information. Cimino et al. [2] describe a prototype WWW-based system providing Internet access to clinical information in a large electronic medical record system. The use of standardized representation models, such as HL7 and SQL, to address the task of integrating legacy clinical information systems with new relational, client-server systems is addressed by Willard et al. [3]. Clemmer [4] challenges us to debate the thesis that proven medical informatics technologies such as electronic medical record systems and clinical reminder tools may have more to offer the emerging field of telemedicine than some of the conventional approaches taken to sup-

\title{
Synopsis
}

\section{Biomedical Information Systems}

port rural and remote practitioners. Banhart et al. [5] discuss the design of an object-oriented system to support the creation of database queries using a sophisticated graphical query generator. Finally, Woelk et al. [6] describe some of the issues faced by our colleagues in developing countries as they begin to apply biomedical information systems technology to publichealth administration.

\section{Clinical Information Systems on the World Wide Web}

The World Wide Web holds much promise as a technology for the development of Internet-based biomedical information systems. It offers a relatively inexpensive, cross-platform, client-server technology for delivering both text and multimedia data over the Internet. Cimino et al. [2] describe a prototype WWW-based system for the retrieval and display of clinical information from a large electronic medical record system. The authors' stated goal is "to develop platform-independent browsing applications that provide health care workers with information about their patients, integrated with access to other medical information sources". The system described in this paper uses an HTML-forms interface which allows the user to create clinical queries. A query is then passed to a set of Common Gateway Interface (CGI) applications running on a WWW server. These CGIs interact with the electronic medical record system to retrieve the desired data and format it as an HTML document which is returned to and displayed within the users' WWW browser application.

The advantages of such a WWWbased clinical information-retrieval system are many. From the users' standpoint it provides an easy-to-use, consistent graphical interface to clinical data that can be used in the hospital, the office or at home. To the biomedical information systems developer it supports rapid prototyping of cross-platform retrieval systems, supporting multimedia and hypertext. In addition, the WWW environment makes it very easy to integrate other Internet-based biomedical information resources.

The authors list a number of potential problems that must be addressed in developing WWW-based clinical information systems. Perhaps the most serious concern is how one maintains the security and confidentiality of patient data that are made available over the Internet. In addition to the standard security features used to protect clinical systems there is a number of emerging Internet security standards, such as Secure Sockets Layer (SSL) and Secure HyperText Transport Protocol (SHTTP) which provide data encryption, server authentication, message integrity, and optional client authentication for the TCP/IP connection underlying the WWW. In addition to using the security features provided by commercial WWW client-server 
systems, the authors also implemented a session key that places time limits on user sessions and implements automatic logout if there is no client activity for a specified time period.

In the conclusion section of their paper the authors raise the interesting prospect that the HTML standard may foster collaborative, rather than competitive research activities. The WWW possesses many attributes that would make such efforts possible, and this technology may be widely used for the integration of clinical information both within and between health-care organizations.

\section{Integrating Legacy Systems into the Client-Server World}

The client-server computing model is becoming the de facto standard for new network-based biomedical information systems. However, many existing legacy systems cannot be replaced with client-server database systems either because of cost or because no appropriate systems exist. The paper by Willard et al. [3] describes the use of a multi-layered connection architecture to feed information from a legacy Laboratory Information System (LIS) to a new network-based client-server, relational database system. The authors offer in the introduction to this paper a number of justifications for moving clinical information systems to the client-server model. Perhaps the most persuasive is that the widespread use of SQL as a query language in this model represents a significant step towards open systems, with the ability to share data across multiple systems. Another suggested advantage is the ability to create client applications with sophisticated graphical user interfaces that may improve ease-of-use and support graphical display of information.

The authors describe a clinical workstation application that they developed and that integrates laboratory data with real-time clinical decision-support tools. In order to allow this client application to access data stored on a legacy LIS, the authors developed a multi-layered architecture using SQL and HL7. Laboratory data are transferred via TCP/IP, in HL7 format, between the LIS and the SQL DBMS, using an Object HL7 transaction/request server. The verified data received from the LIS via this server are duplicated into an SQL environment where external clients may execute queries. To support the kinds of aggregate queries across patient, test and time that many clinical users require, the authors used table-driven remapping of LIS test names to standardized name formats. The authors describe strategies for handling data integrity and synchronization problems that may arise as data are requested via the HL7 server.

The SQL layer satisfies the need for both fixed and ad hoc query capabilities by custom client applications and SQL-compliant commercial products. The authors note that server performance using this approach is acceptable. They also note that the use of standards, such as HL7 and SQL, have allowed them to link to other systems including a WWW-based client for accessing clinical laboratory information. The paper ends with a review of some related strategies for mapping data from legacy systems into relational and object-oriented databases models. The authors suggest that $\mathrm{Ob}-$ ject Database Management Systems (ODBMS) "almost certainly represent the successors to today's SQL servers...but that these databases are still relatively immature compared to their SQL counterparts".

\section{Medical Informatics and Telemedicine}

Clemmer [4] believes that the real impact of medical informatics on telemedicine will be realized by implementing sophisticated clinical information systems with automated reminder and decision-support tools in the rural/remote practices most often targeted by telemedicine initiatives. The author lists the most common perceptions of telemedicine as: (1) Tele-education of providers, administrators and patients; (2) Tele-consultation to rural or remote providers; (3) Tele-patient consultation in which distant consultants can interview and examine patients at remote sites; and (4) Facilitating access to medical literature. He presents arguments why many of these goals are difficult to achieve and may not meet the actual needs of the remote provider and suggests, instead, that we should give priority to implementing computer-based technologies, such as clinical reminder systems that have been demonstrated to enhance patient care in large urban centers.

Most of this paper is a review of how various computer-based clinical decision-support tools can helpus meet our goals of improving the quality of patient care. The author describes datadriven automatic clinical alert systems, such as those implemented in the HELP system [7], and how these tools can effectively deal with large interrelated data sets that tend to overwhelm the average clinician. He surveys computer-based methods for assuring quality of care, monitoring the effectiveness of care, and improving the quality of patient care. In each of these categories he makes persuasive arguments (with references to support them) that medical informatics can significantly enhance these efforts.

The synthesis of these two topics (i.e., effective telemedicine and medical informatics systems that have been shown to improve patient care) is problematic. The conclusion that providing electronic medical record systems with sophisticated reminder tools to 
"even the smallest community hospital or physician's office" is difficult to disagree with but must wait until such time as we solve the technical, administrative and financial problems related to national or regional electronic medical record systems. In the USA the rapid evolution to the managedcare model based on large regional Health Maintenance Organizations may prove to be a stimulus to this process. In the interim this paper provides considerable "food for thought" as to where medical informatics might play an important role in improving patient care, and how it can support the goals of the evolving field of Telemedicine.

\section{Graphical Query Generators}

As we move towards larger clinical and research databases using complex data structures the need to provide easy-to-use, interactive tools for building database queries becomes increasingly important. Banhart et al. [5] describe a graphical query generator for use with a clinical research database. This system was designed to address limitations of the Query by Example (QBE) and SQL tools, most often used with large relational databases. The authors believe that "graphical query languages are eithervery restricted in their features or provide only a low level of abstraction with respect to the underlying logical query language". To address these and other issues the authors developed "a graphical tool for easy generating of even highly complex database queries and for interactive browsing in the database... using a graphical abstraction of a data model with object-oriented features". The paper describes this object-oriented model and the use of this model for the design of a graphical query generator.

Database queries are intrinsically linked to the underlying data model used in a database. Relational models may present problems when one wishes to represent the complex semantic relationships of medical data. Objectoriented data models can represent these types of relationships and the authors describe such a data model as a view of an underlying relational database. This view is implemented as a meta-database projected by an object server consisting of a set of object classes, defining the common behavior of persistent objects and relationships. This object model uses the Entity, Relationships and Attributes(ER) schema to describe the underlying normalized data model. On top of this ER layer, the authors have implemented additional features to support the modeling of user-defined database views using view objects, derived attributes, derived relationships and complex relationships.

View objects define a set of mutually related entities which represent user-specified database views with SQL-based rules defining component relationships. Attributes describe the static properties of entities within a view. These properties consist of machine-level representation (data type) and a higher level display representation (presentation type) which support the mapping of entities from database to graphical interface and abstract the internal representation of data from its graphical representation and behavior as presented to the user. Attributes can also be derived at run-time using embedded SQL code.

Object relationships establish connections between two instances of the same or different object classes and can be represented in graphical environments such as graphs. As with view objects, relationships may be static or derived. Object-oriented data models also support inheritance, and the authors describe two types of inheritance they have implemented: simple and propagated specialization. The latter type of inheritance seeks to address the heterogeneous nature of data stored in large research databases by making it possible to manipulate or hide confounding object attributes.

The query generator described in this paper uses a graphical abstraction of the underlying data model to facilitate an interactive, visual interface to the data entities contained in the database. The authors describe the behavior and properties of this graphical query-creation environment. Objects can be selected and interconnected allowing the user to assemble a graphical view of the data schema for inclusion in a database query. This graphically assembled scheme is then transformed into an SQL "select" statement which is transmitted to the database server. The result of a query is a set of objects which is assembled into a table-based report view containing attributes of both the objects retrieved and selected objects to which they are related.

The authors discuss an evaluation of this system with a database of clinical and genetic data for approximately 700 patients with Retinitis Pigmentosa. They believe that the graphical query generator proved to be a very helpful tool. They state that it was successfully used by physicians, medical students and clinical researchers to search for phenotype data and perform complex statistical analysis. They conclude that "with our graphical user interface even highly complex queries normally generated by experienced database programmers can be composed by non-expert database users".

\section{Creating Health Information Systems in Developing Countries}

Data to support the management process in health care is particularly important in developing countries where there is a need to maximize the use of very limited resources. Woelk 
et al. [6] describe their experience in creating such a system to support the City Health Department of Harare, the capital of Zimbabwe. The Health Department in this city of 1.1 million (1992) has a network of primary care and maternity clinics referring to government-run hospitals. In 1986, the City Health Department began a process to computerize service statistics, beginning with outpatient clinic visits, and this paper reports subsequent developments.

A steering committee of interested municipal representatives was established to begin feasibility studies and select appropriate hardware and software. A plan was formulated and presented to the city but, unfortunately, funding was not available to support the proposed system. With admirable determination a successful attempt was made to use part of the computing system of the municipal Treasury Department to store at least the outpatient-clinic statistics. Following this experiment an IBM PC AT microcomputer with 20 megabytes of disk storage was obtained, and using an inexpensive commercial relational database manager and the Center for Disease Control's EPIINFO software, work was begun on recording most of the other service statistics needed by the Health Department. Attempts to develop custom software for a pharmacy service system were suspended because of anticipated conflicts with the manual methods in use at the time.

The authors make the point that careful planning and establishment of a steering committee allowed them to successfully achieve many of their data-management goals using alternative solutions. The use of existing computer resources, implementation of a training program for those responsible for entering data to the system, ongoing monitoring and consultation with end users and careful control of costs are all identified as important components in their success. The authors state that perhaps the most transparent use of computerization has been to support the process of decentralization and to allow disaggregated health data to be available when needed for local planning, monitoring and decision making activities. It is their belief that this model results in more effective primary health care delivery.

\section{References}

1. Lowe HJ, Lomax EC and Polonkey SE. The World Wide Web: A Review of an Emerging Internet-Based Technology for the Distribution of Biomedical Information. J Am Med Inform Assoc 1996;3:1-
14.

2. Cimino JJ, Socratous SA and Clayton PD. Internet as Clinical Information System: Application Development Using the World Wide Web. J Am Med Inform Assoc 1995;2:273-84.

3. Willard KE, Sielaff BH and Connelly DP. Integrating Legacy Laboratory Information Systems into a Client-Server World: The University of Minnesota Clinical Workstation CWS) Project. Meth Inform Med 1995;34:289-96.

4. Clemmer TP. The Role of Medical Informatics in Telemedicine. $J$ Med Systems 1995;19:47-58.

5. Banhart $F$ and Klaeren $H$. A Graphical Query Generator for Clinical Research Databases. Meth Inform Med 1995;34:32839.

6. Woelk GB and Moyo IM. Development of a Computerized Information System in the Harare City Health Department. Meth Inform Med 1995;34:297-301.

7. Bradshaw KE, Gardner RM, Pryor TA. Development of a Computerized Laboratory Alerting System. Comp Biomed Res 1989;22:575-87.

Address of the author:

Henry J. Lowe, M.D., Section of Medical Informatics, University of Pittsburgh,

B50A Lothrop Hall, Pittsburgh, PA 15261, USA.

E-mail: lowe@nes.nlm.nih.gov http://www.cml.upmc.edu/ImageEngine/ HenryLowe.html 